\title{
A study of the association of glutathione S-transferase M1/T1 polymorphisms with susceptibility to vitiligo in Egyptian patients ${ }^{*}$
}

\author{
Dalia Gamal Aly ${ }^{1}$ \\ Khalda Sayed Amr ${ }^{3}$
}

\author{
Samar Abdallah Salem ${ }^{2}$ \\ Mahmoud Fawzy Abd El-Hamid ${ }^{1}$
}

DOI: http:/ / dx.doi.org/10.1590/abd1806-4841.20185796

\begin{abstract}
BACKGROUND: The association of glutathione S-transferases M1/T1 (GSTM1/T1) null polymorphisms with vitiligo was proposed in several studies including two Egyptian studies with contradictory results.

ОвлЕстIVE: The aim here was to assess the association between GSTM1/T1 null polymorphisms and the susceptibility to vitiligo in a larger sample of Egyptian patients with generalized vitiligo.

MethoDs: This study included 122 vitiligo patients and 200 healthy controls that were age, and gender matched. Assessment of GSTM1/T1 gene polymorphisms was done using a multiplex polymerase chain reaction (PCR).

RESULTS: Increased odds of generalized vitiligo was observed with the null genotypes of GSTM1 and GSTT1 polymorphisms $(\mathrm{P}<0.05)$. Controls with GSTM1 null/GSTT1+ heterozygosis presented with a 2.97 odds protection from having generalized vitiligo $(\mathrm{OR}=2.97,95 \% \mathrm{CI}=1.1-7.7)(\mathrm{P}=0.02)$ compared with patients.

STUDY LimitATIONS: Small sample size of patients.

CONCLUSIONS: This study showed a significant trend towards an association with the combination of the GSTM1/GSTT1 double null polymorphism and generalized vitiligo. Individuals with GSTM1 null/GSTT1+ heterozygosis have a 2.97 odds protection from having generalized vitiligo compared with patients. It was is the first time, to our knowledge, that such an association has been reported.
\end{abstract}

Keywords: Glutathione S-Transferase pi; Oxidative stress; Polymorphism, single nucleotide; Vitiligo

\section{INTRODUCTION}

Vitiligo is a skin disease related to unidentified environmental triggers and numerous susceptibility genes. Candidate gene linkage and genetic association reports have involved a number of possible contributory loci, although there is little data. ${ }^{1}$ Multiple factors and overlapping pathogenetic mechanisms are suggested for the disorder; however, the cellular mechanisms resulting in the loss of functional epidermal melanocytes is still obscure. ${ }^{2}$ Increasing evidence indicates that oxidative stress is considered to be one of the possible pathogenic events in melanocyte degeneration. An increased intracellular production of reactive oxygen species due to mitochondrial impairment and a compromised antioxidant status can lead to cellular disruption and consequently, vitiligo. ${ }^{3}$

Members of the glutathione S-transferases (GSTs) supergene family are known to have a vital role in the detoxification of several endogenic oxidative stress products and exogenic carcinogens. ${ }^{4}$ In humans, GST family comprises numerous microsomal, cytosolic and mitochondrial proteins. Eight evident classes have been attributed to the cytosolic family: alpha, kappa, mu, omega, pi, sigma, theta, and zeta. The GST mu class, coded by the GSTM1 gene, is situated in the short arm of chromosome 1 (1p13.3). ${ }^{5}$ The GST theta class, coded by the GSTT1 gene, is situated on the long arm of chromosome 22 (22q11.23). ${ }^{6}$ Glutathione S-transferase M1 and GSTT1 gene demonstrate an inherited homozygous deletion polymorphism (null genotype) that is linked to lack of enzyme activity. A heightened risk to diseases related to oxidative stress as malignancies, respiratory and cardiovascular disorders is found in individuals with homozygous deletion polymorphism. ${ }^{2}$

\section{Received on 18.03.2016.}

Approved by the Advisory Board and accepted for publication on 28.06.2016.

* Work performed at the Department of Dermatology and Venereology, National Research Centre - Department of Medical Molecular Genetics, National Research Centre, Giza, Egypt. Department of Dermatology, Venereology and Andrology, Ain Shams University, Abbasseya Square - Cairo, Egypt. Financial support: None.

Conflict of interest: None.

\footnotetext{
Department of Dermatology and Venereology, National Research Centre - Giza, Egypt.

Department of Dermatology, Venereology and Andrology, Ain Shams University - Cairo, Egypt.

Department of Medical Molecular Genetics, National Research Centre - Giza, Egypt.
}

\author{
MAILING AdDRESS: \\ Dalia Gamal Aly \\ E-mail: dalia.g.aly@gmail.com
}


In 2007, Uhm et al first investigated the relationship between the risk of vitiligo and its association to GSTM1 and GSTT1 gene polymorphisms. Subsequently, several epidemiological studies have aimed to evaluate this association in diverse ethnicities, including two studies performed with an Egyptian population..$^{8-11}$ However, no clear consensus was reached. The studies until now performed with Egyptian patients besides being very scarce, had contradictory results. In order to provide better evidence of such association with vitiligo risk in Egyptian patients, we further evaluated GSTM1 and GSTT1 gene polymorphisms in vitiligo susceptibility in a relatively larger number of patients than those reported in the two previous studies.

\section{METHODS}

We conducted a case-control study on 122 Egyptian patients (89 females and 33 males) with generalized vitiligo. Activity of vitiligo was based on the emergence of new areas of disease, or the enlargement of previous lesions in the last 3 months prior to presentation. ${ }^{12}$ As controls, two hundred (142 females and 58 males) age and sex matched healthy volunteers, who did not have any autoimmune disorders nor any personal or family history of vitiligo were analyzed. All enrolled individuals were selected from the Dermatology outpatient clinics of the National Research Centre and Ain Shams University hospital. All individuals signed an informed consent before contributing in this study. An approval for this work was obtained by the ethical committee of the National Research Centre, Giza, Egypt.

\section{Analysis of GSTM1 and GSTT1 polymorphisms}

Genomic DNA was prepared from peripheral blood lymphocytes from all subjects using QIA amp DNA, mini Kit (QIAGENE Inc., Germany). The GSTM1 and GSTT1 polymorphisms were genotyped by multiplex PCR using the $\beta$-globin gene as a positive control for amplification. The presence of GSTM1 and GSTT1 yielded specific products of 215 and $480 \mathrm{bp}$, respectively, in addition to the control $\beta$-globin product which was $268 \mathrm{bp}$. The following primers were used to amplify GSTM1 gene: $5 \backslash$-GAG GAA CTC CCT GAA AAG CTA AAG-3/ (forward) and 5 - CTC AAA TAT ACG GTG GAG GTC AAG-3/ (reverse). The GSTT1 gene was amplified with the following primers: 5/-TTC CCT ACT GGT CCT CAC ATC TC-3 (forward) and 5\-TCA CCG GAT CAT GGC CAG CA-3/ (reverse). The $\beta$-globin gene loci were CAACTTCATCCACGTTCACC and - GAAGAGCCAAGGACAGGTAC. PCR reaction conditions of $25 \mu \mathrm{L}$ were: initial denaturation $94^{\circ} \mathrm{C}$ for $5 \mathrm{~min}$; $35 \mathrm{cy}$ cles of $94^{\circ} \mathrm{C}$ for $30 \mathrm{~s}, 61^{\circ} \mathrm{C}$ for $30 \mathrm{~s}$, and $72^{\circ} \mathrm{C}$ for $30 \mathrm{~s}$; with final extension of $72^{\circ} \mathrm{C}$ for $5 \mathrm{~min}$. Amplified PCR products were analyzed for successful amplification on $2 \%$ agarose gel electrophoresis stained with ethidium bromide.

\section{Statistical analysis}

Analysis of the data was done by the statistical program for social science (SPSS) 15.0.1 for Windows; SPSS Inc, Chicago, IL, 2001. Continuous data were described using mean and standard deviation (SD). Categorical variables were expressed as frequencies and percent. Comparison between two means and more than two means was done using student t-test and ANOVA test respectively. Chi-square test was used when associating two categorical variables while Fisher's exact test was used if the expected count was less than 5 in more than $20 \%$ of cells. Logistic regression was used for calculating odds ratio. P-values $<0.05$ were considered significant in all tests.

\section{RESULTS}

One hundred and twenty-two Egyptian patients with generalized vitiligo were enrolled in the study, 89 (73.0\%) were females and $33(27.0 \%)$ were males. The disease was active in $63.1 \%$ of the patients and was stable in $36.9 \%$. Their ages ranged from $10-71$ years, with a mean \pm SD of $29.8 \pm 12.7$ years. Disease duration was from 0.5 to 11 years with a mean \pm SD of $2.7 \pm 1.8$ years. Family history was positive in $31.0 \%$ of patients.

The control group included 200 subjects, 142 (71\%) females and $58(29 \%)$ males. Their ages ranged from $11-73$ years with a mean \pm SD of $29.8 \pm 13.5$ years. No significance was noticed regarding the age and gender amongst the studied groups ( $\mathrm{P}$-value $>0.05$ ).

Genotype distribution of the GSTM1 and GSTT1 polymorphisms between patients with generalized vitiligo and controls

Regarding the gene polymorphism frequencies in generalized vitiligo, the GSTM1+/null, GSTT1+/null and double null polymorphisms was $47.5 \%, 52.5 \%, 48.4 \%, 51.6 \%$ and $9.8 \%$, respectively. The combined GSTM1 and GSTT1 gene polymorphisms, GSTM1+/GSTT1+, GSTM1-/GSTT1+, GSTM1+/GSTT1-, were $5.7 \%, 42.6 \%$ and $41.8 \%$, respectively; whereas in controls, GSTM1+/ null, GSTT1+ / null and double null was 44.5\%, 55.5\%, 59\%, 41\% and $4 \%$ respectively. The combined GSTM1 and GSTT1 gene polymorphisms, GSTM1+/GSTT1+, GSTM1-/GSTT1+, GSTM1+/GSTT1-, were $7.5 \%, 51.5 \%$, and $37 \%$, respectively (Table 1, Figure 1 ).

Compared to the control group, no association was detected with either GSTM1+ / null nor GSTT1+ / null and generalized vitiligo $(\mathrm{P}>0.05, \mathrm{OR}=0.885,95 \% \mathrm{CI}=0.56-1.3 ; \mathrm{P}>0.05 \mathrm{OR}=1.5,95 \% \mathrm{CI}=0.97-$ 2.4 , respectively).

To evaluate the combined effect of GSTM1 and GSTT1 gene polymorphisms in generalized vitiligo, both present types of GSTM1 and GSTT1 genes as well as GSTM1null/ GSTT1+, and GSTM1+/ GSTT1null were compared to both double null types within the patient group. A significant association of the double null genotype was detected with generalized vitiligo susceptibility $(\mathrm{P}<0.05, \mathrm{OR}=0.382,95 \% \mathrm{CI}=0.15-0.96)$; while GSTM1null/ GSTT1+ was associated with a 2.97 -fold lower odd of having the disease $(\mathrm{P}<0.05, \mathrm{OR}=2.97,95 \% \mathrm{CI}=1.1-7.7)$.

Analyzing the association between GSTM1+/null, GSTT1+/ null gene polymorphisms and the clinical features and family history in patients with vitiligo, no significant relationship was found with either age, disease duration, family history, or disease activity (Table 2). There was also no significant relationship with the combined analysis (P-value>0.05) (Table 3).

Comparison of GST polymorphisms frequencies between males and females

Furthermore, we investigated if the frequencies of GST polymorphisms were different between males and females. The total number of patients who harbored GSTM1+ was 58 [17 (29.3\%) males and $41(70.7 \%)$ females], whereas 64 patients had GSTM1 null genotype [16 (25.0\%) males and 48 (75.0\%) females]. Meanwhile, 59 patients harbored GSTT1+ genotype [16 (27.1\%) males and 43 (72.9\%) females] while 63 patients [17 (27.0\%) males and $46(73.0 \%)$ 


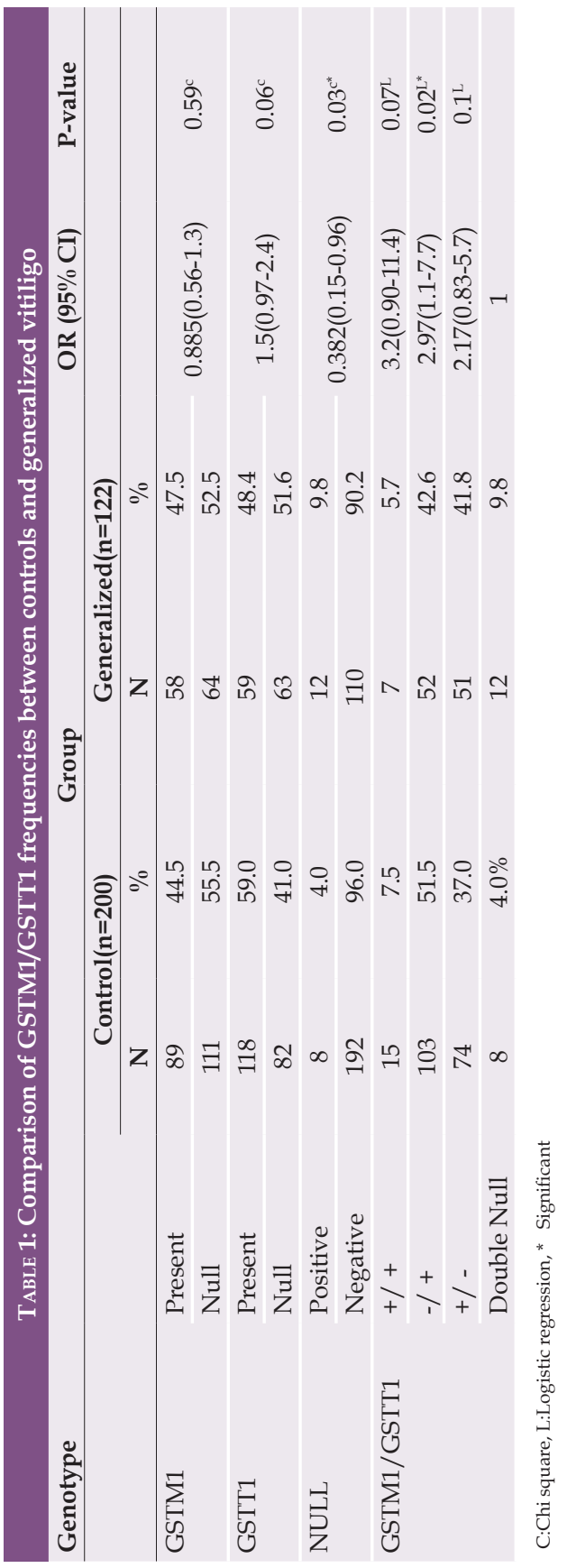

females] had GSTT1 null genotype. No significant difference was detected between males and females as regards the GST polymorphism gene frequencies ( $P$ value $>0.05$ ).

On analyzing the combined effects of GST gene polymorphism in both males and females, we found that the GSTM1+/ GSTT1+ genotype was present in 7 patients [ 4 (57.1\%) males and 3 (42.9\%) females] while GSTM1/GSTT1 double null genotype was present in 12 patients [ $4(33.3 \%)$ males and $8(66.7 \%)$ females]. Moreover, we compared the frequency of GSTM1+/GSTT1+ (both present types) to the frequency of each of GSTM1/GSTT1 double null genotype, GSTM1 null/GSTT1+ and GSTM1+/GSTT1 null in both sexes and noted a non-significant difference between them (P value $>0.05)$.

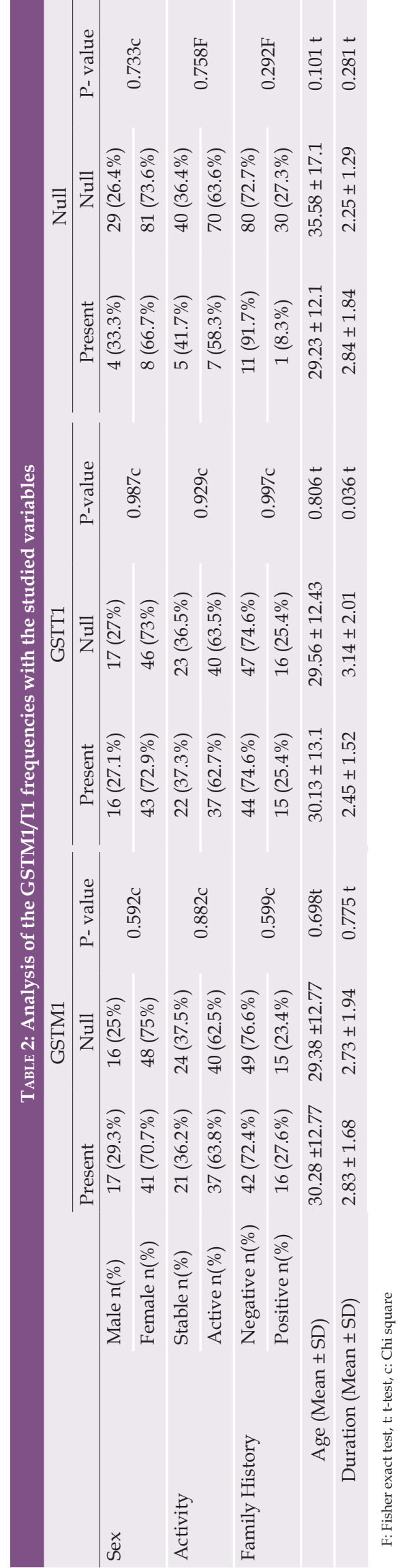




\begin{tabular}{|c|c|c|c|c|c|c|}
\hline & & GSTM1+/GSTT1- & GSTM1-/GSTT1+ & GSTM1+/GSTT1+ & GSTM1-/GSTT1- & $P$-value \\
\hline \multirow[t]{2}{*}{ Sex } & Male $\mathrm{n}(\%)$ & $13(25.5 \%)$ & $12(23.1 \%)$ & $4(57.1 \%)$ & $4(33.3 \%)$ & $0.255 \mathrm{~F}$ \\
\hline & Female $n(\%)$ & $38(74.5 \%)$ & $40(76.9 \%)$ & $3(42.9 \%)$ & $8(66.7 \%)$ & \\
\hline \multirow[t]{2}{*}{ Activity } & Stable $\mathrm{n}(\%)$ & $18(35.3 \%)$ & $19(36.5 \%)$ & $3(42.9 \%)$ & $5(41.7 \%)$ & $0.944 \mathrm{~F}$ \\
\hline & Active $\mathrm{n}(\%)$ & $33(64.7 \%)$ & $33(63.5 \%)$ & $4(57.1 \%)$ & $7(58.3 \%)$ & \\
\hline \multirow[t]{2}{*}{ Family History } & Negative & $36(70.6 \%)$ & $38(73.1 \%)$ & $6(85.7 \%)$ & $11(91.7 \%)$ & $0.5 \mathrm{~F}$ \\
\hline & Positive & $15(29.4 \%)$ & $14(26.9 \%)$ & $1(14.3 \%)$ & $1(8.3 \%)$ & \\
\hline \multicolumn{2}{|c|}{ Age (Mean \pm SD) } & $28.84 \pm 11.82$ & $29.06 \pm 11.42$ & $33.29 \pm 19.15$ & $35.58 \pm 17.1$ & $0.328 \mathrm{~A}$ \\
\hline \multicolumn{2}{|c|}{ Duration (Mean $\pm S D$ ) } & $2.5 \pm 1.58$ & $2.96 \pm 1.74$ & $4.43 \pm 3.36$ & $2.25 \pm 1.29$ & $0.21 \mathrm{~A}$ \\
\hline
\end{tabular}

F: Fisher exact test , A: ANOVA

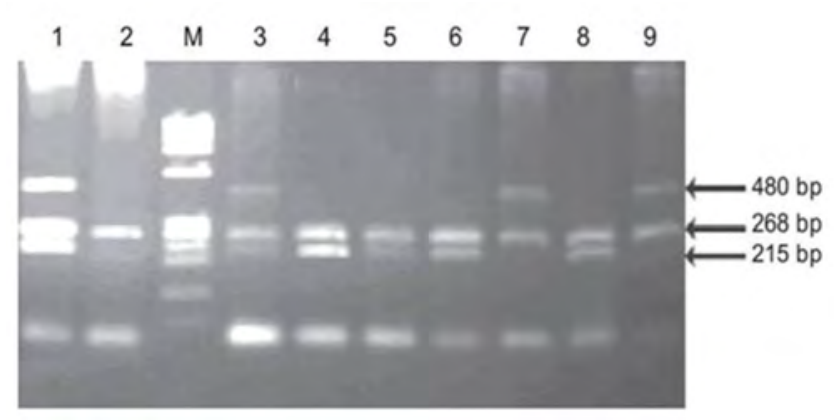

FIGURE 1: Agarose gel electrophoresis of PCR amplification products. The $480 \mathrm{bp}$ band corresponds toGSTT1, $268 \mathrm{bp}$ to $\beta$-globin and 215 bp to GSTM1. Lanes 1 and 3: GSTT1 positive/GSTM1 positive. Lanes 4, 5,6 and 8: GSTM1 positive/GSTT1 null. Lanes 7 and 9: GSTT1 positive/GSTM1 null. Lane2: GSTM1 null/GSTT1 null .M: px 174/HaeIIII ladder

\section{DISCUSSION}

The objectives of this study were to analyze the frequency of GSTM1/T1 genotypes and to determine whether a GST polymorphism is a predisposing factor to generalized vitiligo. Furthermore, we investigated the hypothesis presented by "Bassiouny and Khorshied", 2013"11 that Egyptian patients with vitiligo who demonstrate the GSTT1/GSTM1 double null polymorphism together with the absence of the GSTM1 gene are at increased risk of having the disease.

In the total of 122 patients enrolled in this study, the frequency of the GSTM1/GSTT1 double null genotype was $9.8 \%$ while the frequency of GSTM1 null genotype was 52.5\% and that of GSTT1 null genotype was 51.6\%. The previous Egyptian studies done on vitiligo patients demonstrated $18.8 \%$ for GSTM1/T1 double null genotype, $56.4 \%$ for GSTM1 null and 30.7\% for GSTT1 null11 and 30\% for GSTM1/T1 double null genotype, 52.5\% for GSTM1 null and $57.5 \%$ for GSTT1 null. ${ }^{10}$

The differences in the rates of GST double null genotype and GSTT1 null between our study and the previous Egyptian studies could be attributed to ethnicity. The ethnic origin of Egyptians ought to be determined in GST gene polymorphisms studies. In a study performed by Nelson et al in $1995^{13}$ on five different ethnic groups, there were also significant discrepancies in the frequency of GSTT1 null genotype among the Caucasian population and suggested that the ethnic origin in Caucasians must as well be determined in reports of gene-environment interplay related to this polymorphism.
Moreover, we evaluated if the percentage of deletions found in this work were different in males than females. The percentage of cases with the 'double null' genotype was higher in females $[66.7 \%$ $(8 / 12)]$ than in males [33.3\% (4/12)]. Equivalently, the percentage of the GSTM1 null genotype was greater $(75 \%)$ in females $(48 / 64)$ than in males [25\% (16/64)], whereas the GSTT1 null genotype was also higher $(73 \%)$ in females $(46 / 63)$ than in males [27\% (17/64)]. Ultimately, the percentage of cases with no deletion perceived (both genes present) was higher in males [57.1\% (4/7)] than in females [(42.9\%) (3/7)].

We were not able to compare our results to the two previous Egyptian reports as, Abd Rabou et al, in 2011[10], did not report such a comparison whereas Bassiouny and Khorshied, in 2013 ${ }^{11}$, performed their study with female patients only. Whether our results denote that males and females have different underlying mechanisms leading to their risk of vitiligo, or that it is due to a bias in the selection of cases, or that there was a relatively limited number of patients, is not apparent. Nevertheless, these findings indicate that attention is needed before recruiting males and females for assessing GST polymorphism genotypes as a predictor of vitiligo risk. ${ }^{14}$

On assessing the association of GSTM1 and GSTT1 polymorphism with generalized vitiligo, we noted that there were no differences between either GSTM1+/null or GSTT1+/null polymorphism between the studied groups, and that none of them had any significant association with vitiligo susceptibility. However, a notable association to the risk of vitiligo was found with the GSTM1/GSTT1 double-null type polymorphism. Moreover, controls with GSTM1 null/GSTT1+ heterozygosis presented with a 2.97 odds protection from having generalized vitiligo compared with patients (a finding that contradicts the two previous Egyptian studies). Our findings are in agreement with former studies in the literature associating the double-null genotype with a substantially heightened vitiligo risk. ${ }^{7-11}$ Yet, there are discrepancies in the association of vitiligo with one GST null allele.

Uhm et al, in $2007^{7}$, were the first to reveal that GSTM1 null or GSTM1/ T1-double null polymorphism could be associated with the risk of vitiligo in the Korean population, but they did not find the difference in the GSTT1 null polymorphism between the studied groups. Similarly, Lu et al, in $2014^{15}$, also reported no diversity in GSTT1 null polymorphism within patients and controls in Chinese individuals; the results involving the GSTM1 null and GSTM1/ T1-double null polymorphism was consistent with the study by Uhm and his colleagues. However, another study by Liu et al, 20098, showed that either GSTT1 null, GSTM1 null or GSTM1/T1 double null polymorphism might increase the susceptibility to vitiligo in 
the Chinese population. The inconsistent results of GST polymorphisms between the former three studies and our study come from the differences in sample sizes as well as the heterogeneity of the populations studied. ${ }^{15}$

Meanwhile, in two studies carried out on Mediterranean population, Guarneri et al, 2011 ${ }^{[9]}$ (Italian) and Bassiouny and Khorshied, 201311, found that GSTM1 null or GSTM1/T1 double null polymorphism might increase the risk of vitiligo. Interestingly, the study by Abd Rabou et al in $2011^{10}$, also suggested that GSTM1/ T1 double null polymorphism was related to the risk of vitiligo in the Egyptian population, but they did not find the difference in the polymorphisms of GSTM1 null between cases and controls. Our results disagree with Bassiouny and Khorshied, $2013^{11}$, as we did not find any association of vitiligo with GSTM1 null genotype. We believe that the difference between their findings and ours might come from their smaller sample size of subjects involved than our study (101 female patients and 101 controls) as well as patient selection (only females were involved). Despite the fact that the sample size was also small in the study of Abd Rabou and his colleagues, which included 40 patients with different types of vitiligo and 10 subjects in the control group, that consequently had inevitably limited results; yet, our findings are in agreement with them.

The prophylactic role of antioxidant enzymes is through neutralizing ROS and different toxic substances, hence hindering their resultant toxic effects and their melanocyte accumulation. An individual's redox homeostasis depends on the activity of a given GST isoform; having the correspondent null allele might considerably heighten the chance of having vitiligo, whilst the GSTM1/GSTT1 double-null genotype consistently raises such a risk due to the significant reduction in the basal antioxidant potential of me- lanocytes (caused by the simultaneous lack of GSTM1 and GSTT1) and the incapability to upregulate the expression of GSTM1/GSTT1 in response to oxidative stress. ${ }^{9}$

Differences between the studies propose that the importance and the role of each GST isoform in the pathogenesis of vitiligo are inconsistent, and are perhaps associated with numerous aspects such as evolutionary choice and adjustment to environmental circumstances. Accordingly, several patterns of the system are prone to be present in diverse populations. ${ }^{11}$

In this study we also speculated that if a specific GST polymorphism can identify the activity of vitiligo, then it is probable that treatment of such patients could be modified to improve their prognosis. Our observations of no significant differences in the GST polymorphisms as regards disease activity propose that either the inherited absence or presence of the detoxification pathway of GSTT1 and GSTM1 is not a predisposing factor involved in the prognosis of vitiligo. We also found no interaction with age, disease duration, or family history of patients.

\section{CONCLUSION}

Our findings enlighten the relations between vitiligo, oxidative stress, and genetics. Overall the present study showed a significant trend towards an association with the combination of the GSTM1/GSTT1 double null polymorphism and generalized vitiligo. Individuals with GSTM1 null/GSTT1+ heterozygosis have a 2.97 odds protection from having generalized vitiligo. To our knowledge, it is the first time that such an association is reported in Egyptian patients. $\square$

\section{REFERENCES}

1. Jin Y, Birlea SA, Fain PR, Gowan K, Riccardi SL, Holland PJ, et al. Variant of TYR and autoimmunity susceptibility loci in generalized vitiligo. N Engl J Med. 2010;362:1686-97

2. Gao LB, Pan XM, Li LJ, Liang WB, Bai P, Rao L, et al. Null Genotypes of GSTM1 and GSTT1 Contribute to Risk of Cervical Neoplasia: An Evidence-Based MetaAnalysis. PLoS One. 2011:6:e20157.

3. Shin JW, Nam KM, Choi HR, Huh SY, Kim SW, Youn SW, et al. Erythrocyte malondialdehyde and glutathione levels in vitiligo patients. Ann Dermatol. 2010;22:279-83.

4. Ketterer B. Protective role of glutathione and glutathione transferases in mutagenesis and carcinogenesis. Mutat Res. 1988;202:343-61.

5. Pearson WR, Vorachek WR, Xu SJ, Berger R, Hart I, Vannais D, et al. Identification of class-mu glutathione transferase genes GSTM1-GSTM5 on human chromosome 1p13. Am J Hum Genet. 1993:53:220-33.

6. Webb G, Vaska V, Coggan M, Board P. Chromosomal localization of the gene for the human theta class glutathione transferase (GSTT1). Genomics. 1996;33:121-3.

7. Uhm YK, Yoon SH, Kang IJ, Chung JH, Yim SV, Lee MH. Association of glutathione S-transferase gene polymorphisms (GSTM1 and GSTT1) of vitiligo in Korean population. Life Sci. 2007;81:223-7.

8. Liu L, Li C, Gao J, Li K, Gao L, Gao T. Genetic polymorphisms of glutathione $\mathrm{S}$-transferase and risk of vitiligo in the Chinese population. $\mathrm{J}$ Invest Dermatol. 2009;129:2646-52.
9. Guarneri F, Asmundo A, Sapienza D, Cannavò SP. Glutathione S-transferase M1/ T1 gene polymorphisms and vitiligo in a Mediterranean population. Pigment Cell Melanoma Res. 2011;24:731-3.

10. Abd Rabou F, Elserogy H, Gheida S, El-Ashmawy A. Glutathione S-transferase gene polymorphisms (GSTM1 and GSTT1) in vitiligo patients. Life Sci J 2011; 8:785-792.

11. Bassiouny DA, Khorshied MM. Glutathione S-transferase M1 and T1 genetic polymorphism in Egyptian patients with nonsegmental vitiligo. Clin Exp Dermatol. 2013;38:160-3.

12. Li M, Gao Y, Li C, Liu L, Li K, Gao L, et al. Association of COX2 functional polymorphisms and the risk of vitiligo in Chinese populations. J Dermatol Sci. 2009;53:176-81.

13. Nelson HH, Wiencke JK, Christiani DC, Cheng TJ, Zuo ZF, Schwartz BS, et al. Ethnic differences in the prevalence of the homozygous deleted genotype of glutathione S-transferase theta. Carcinogenesis. 1995;16:1243-5.

14. Sheikhha MH, Kalantar M, Tobal K , Liu Yin JA . Glutathione S-transferases Null Genotype in Acute Myeloid Leukaemia. IJI. 2005;2:141-51.

15. Lu L, Wu W, Tu Y, Yang Z, He L, Guo M. Association of glutathione S-transferase M1/T1 polymorphisms with susceptibility to vitiligo. Gene. 2014;535:12-6.

How to cite this article: Aly DG, Salem SA, Amr KS, Abd El-Hamid MF. A study of the association of glutathione S-transferase M1/T1 polymorphisms with susceptibility to vitiligo in Egyptian patients. An Bras Dermatol.2018;93(1):54-8. 